\title{
Modeling the Surround of MT Cells and Their Selectivity for Surface Orientation in Depth Specified by Motion
}

\author{
Lin Liu \\ Marc M. Van Hulle \\ K.U. Leuven, Laboratorium voor Neuro- en Psychofysiologie, Leuven, Belgium
}

The projective transformation onto the retina loses the explicit $3 \mathrm{D}$ shape description of a moving object. Theoretical studies show that the reconstruction of 3D shape from 2D motion information (shape from motion, SFM) is feasible provided that the first- and second-order directional derivatives of the 2D velocity field are available. Experimental recordings have revealed that the receptive fields of the majority of the cells in macaque area middle temporal (MT) display an antagonistic (suppressive) surround and that a sizable portion of these surrounds are asymmetrical. This has led to the conjecture that these cells provide a local measure for the directional derivatives of the $2 \mathrm{D}$ velocity field. In this article, we adopt a nonparametric and biologically plausible approach to modeling the role played by the MT surrounds in the recovery of the orientation in depth (the slant and tilt) of a moving (translating) plane. A three-layered neural network is trained to represent the slant and tilt from the projected motion vectors. The hidden units of the network have speed-tuning characteristics and represent the MT model neurons with their surrounds. We conjecture that the MT surround results from lateral inhibitory connections with other MT cells and that populations of these cells, with different surround types, code linearly for slant and tilt of translating planes.

1 Introduction

There is a growing body of evidence that the middle temporal (MT, V5) cortical area, located in the posterior bank of the superior temporal sulcus, systematically represents the direction and velocity of visual motion (Allman \& Kaas, 1971; Dubner \& Zeki, 1971; Albright, 1984; Maunsell \& Van Essen, 1983; Movshon, Adelson, Gizzi, \& Newsome, 1985; Lagae, Raiguel, \& Orban, 1993). More than $90 \%$ of the MT neurons have a surround that envelops the classical receptive field (CRF) (Allman, Miezen, \& McGuinness, 1985), and it reveals its presence only by the effect on the CRF response. Since the MT surrounds are extensive in size, it is believed that MT neurons are able to integrate local stimulus conditions within a global context (localto-global comparison) (Allman et al., 1985). Furthermore, the majority of 
the MT surrounds in the macaque monkey are antagonistic (suppressive) (Raiguel, Van Hulle, Xiao, Marcar, \& Orban, 1995; Tanaka et al., 1986). This has led to a series of speculations about the role of MT surrounds: figureground segregation, preattentive vision, depth perception, detection of motion discontinuities, and differentiation of object motion from egomotion (Albright, 1984, 1993; Allman et al., 1985; Tanaka et al., 1986).

Lesion studies performed by Andersen and Siegel (1986) have shown that MT plays a prominent role in the perception of 3D shape in moving stimuli (shape from motion, SFM). Furthermore, evidence indicating that human SFM perception involves surface interpolation (Hussain, Treue, \& Andersen, 1989; Treue, Andersen, Ando, \& Hildreth, 1995) has led to the hypothesis that the spatial structure of the receptive field (RF) is involved in this process. Hence, in this view, SFM is redefined as the problem of characterizing interpolating surfaces. Spurred by the progress in optic flow theory, physiological predictions were formulated about the RF profiles needed to allow for an SFM extraction (Droulez \& Cornilleau-Pérès, 1990; Koenderink \& van Doorn, 1992) but without making clear statements whether these models applied to MT neurons. Buračas and Albright (1994) have modeled the velocity selectivity curves of MT neurons as sums and differences of two gaussian-distributed velocity filters: one for the center and another for the surround (a parametric model). However, they did not model the spatial layout of the MT RF itself and assumed it to be radially symmetrical, but they also qualified their assumption by predicting the existence of asymmetrical surrounds (but, "possibly in areas FST or MST," p. 975).

Recently, it was found that the majority of the MT surrounds are not radially symmetrical (Xiao, Raiguel, Marcar, Koenderink, \& Orban, 1995, 1997; Xiao, Marcar, Raiguel, \& Orban, 1997), in contrast with what was previously assumed (Tanaka et al., 1986). The type of surrounds obtained led the experimentalists to conjecture that MT could locally compute the firstand second-order directional derivatives of the velocity field induced by the moving (translating) object (Xiao et al., 1995). Such a conjecture has important implications since theoretical studies show that SFM is feasible when these directional derivatives are available (Droulez \& CornilleauPérès, 1990; Koenderink \& van Doorn, 1992). Inspired by this new experimental evidence, Buračas and Albright (1996) introduced asymmetrical surrounds in their parametric model and discussed the implication on SFM extraction. Finally, we should add that to date, no published experimental study has demonstrated selectivity for surface curvature in single MT cells; existing studies have looked for orientation in depth selectivity (slant and tilt selectivity) of translating planes (Treue et al., 1995; Xiao et al., 1995, 1997). (In addition to curvature, slant and tilt are commonly used geometrical descriptors of surface shape.)

In order to investigate the putative contribution of MT cells to SFM, and in particular to the recovery of the slant and tilt of a translating plane, we develop in this article a biologically plausible model of MT RFs by using the 
known RF properties for guidance. However, unlike Buračas and Albright (1996), we avoid adopting a parametric approach that considers the MT CRFs and their surrounds as differential motion filters for estimating space differentials from which local surface shape can be determined. Instead, we adopt a nonparametric approach and train a three-layered recurrent network wherein the MT model neurons comprise the hidden units and whose output units represent the desired slant and tilt of the translating plane. Furthermore, since speed tuning is one of the most important properties of MT cells (Lagae, Raiguel, \& Orban, 1993; Maunsell \& Van Essen, 1983), and also of their surrounds (Allman et al., 1985; Tanaka et al., 1986; Lagae, Gulyás, Raiguel, \& Orban, 1989, Xiao et al., 1997), we assume that our hidden units are endowed with a speed tuning characteristic by their input units. The shapes and characteristics of the CRFs and surrounds developed are in accordance with those found in single-cell recordings primarily in four ways: (1) the surrounds of our MT neurons are predominantly antagonistic and reveal their presence only when the CRF is stimulated; (2) the surrounds developed are radially symmetric, bilaterally symmetric, or asymmetric; (3) the axis of the bilaterally symmetric surrounds is orthogonal to the long axis of the elliptically shaped CRF; and (4) the surround inhibition is speed tuned. We conjecture that the surround results from lateral inhibitory connections within MT and that populations of MT cells code linearly for slant and tilt of translating surfaces.

\section{Velocity Gradient as a Cue for Slant and Tilt}

Most computational approaches to SFM are rooted in the observation that the 3D shape of a solid, smooth object can be locally approximated by the first-order geometrical descriptors slant and tilt. We consider a translating plane, on which dots are densely distributed, and project the corresponding motion vectors onto the fronto-parallel plane (orthogonal to the line of sight). Hence, the slant and tilt of the translating surface are the sole variables in our SFM problem, up to a scaling factor, and they are to be determined from the gradient of the projected motion vectors (to a first approximation, the velocity field caused by translational motion of a smooth surface is proportional to the surface shape). As a result of the projective transformation, the observer will have the impression that dots located nearer to him or her are moving faster, while dots farther away are moving more slowly. (Because the surface undergoes only translational motion, we will not distinguish between observer ego motion and surface motion.)

The stimulus setup and coordinate system are shown in Figure 1. The origin $(0,0,0)$ is set at the fixation point on the moving surface. The observer, located at $O(0,0, b)$, is at a distance $b$ from the fixation point. The plane through $R(0,0, a)$, and orthogonal to the z-axis (line of sight), is taken as the projection plane. For a given position $Q(x, y, z)$ on the moving surface, the line $O Q$ intersects the projection plane in $P\left(x_{0}, y_{0}, a\right)$. (The arbitrary choice of 


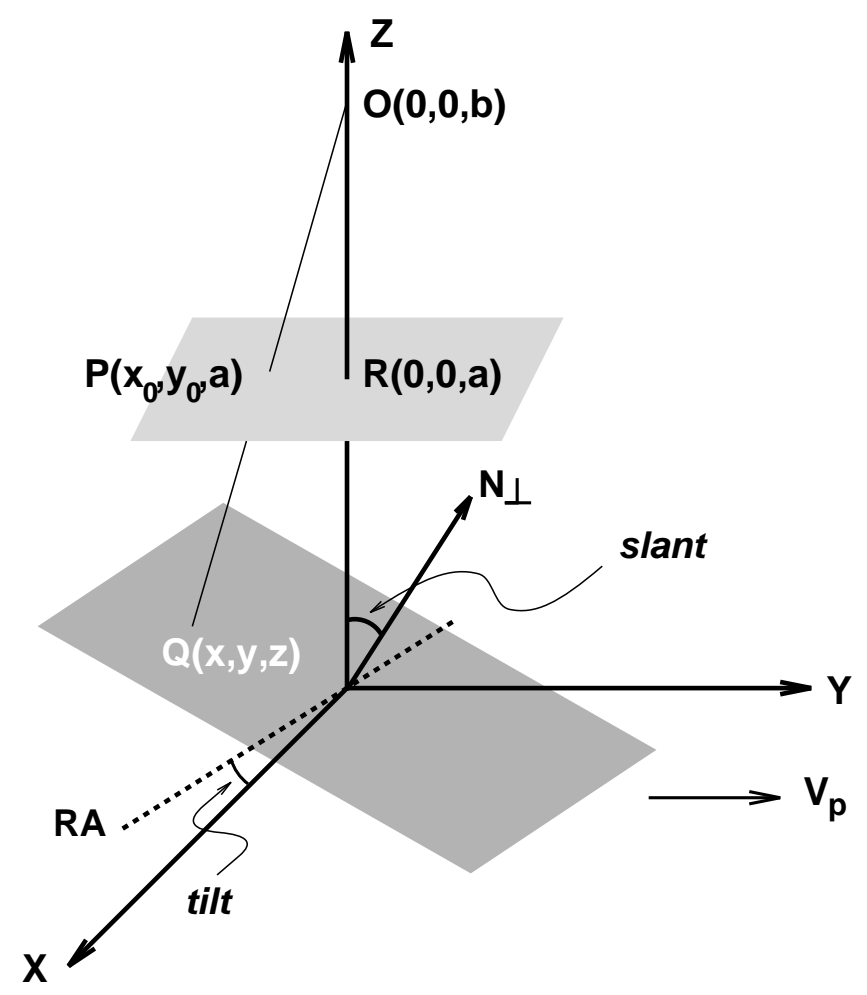

Figure 1: The stimulus setup and coordinate system used. The observer is at point $O$, and the fixation point, on the moving plane, is at the origin of the coordinate system $X Y Z$. The line of sight corresponds to the z-axis. The line $O Q$, with point $Q$ on the moving plane, intersects at point $P$ on the projection plane. The projection plane is orthogonal to the line of sight and fixed at a distance $a$ from the fixation point. The slanted and tilted plane undergoes translational motion in the direction of the y-axis with velocity $V_{p}$. The slant and tilt, as well as the inward surface normal $N_{\perp}$, are indicated. The axis $R A$ (dashed line) is needed in the definition of slant and tilt.

the line of sight implies that all computations are supposed to be local.) The slant and tilt are defined as follows. Consider first the moving surface to be parallel to the projection plane and define an axis $R A$, on the moving surface, that at first coincides with the $\mathrm{x}$-axis. The slant $\theta$ is the angle defined by a rotation of the moving surface around $R A$. The tilt $\alpha$ is the angle defined by a rotation of $R A$ itself around the $\mathrm{z}$-axis. (Note that the slant also corresponds to the angle between the line of sight and the inward surface normal $N_{\perp}$; the tilt also corresponds to the angle between the y-axis and the direction 
of decreasing distance.) The parameter equation of the line $O P Q$ is:

$$
\left\{\begin{array}{l}
x=x_{0} t \\
y=y_{0} t \\
z=b+(a-b) t
\end{array}\right.
$$

where, for point $P t=1$, and for point $Q$ :

$$
t=\frac{b \cos \theta}{x_{0} \sin \alpha \sin \theta+y_{0} \cos \alpha \sin \theta-(a-b) \cos \theta} .
$$

Assuming that the plane moves with velocity $V_{p}$ in the direction of the y-axis, the velocity $V$ at point $P\left(x_{0}, y_{0}\right)$, in the projection plane, is then:

$$
V\left(x_{0}, y_{0}\right)=V_{p} \frac{x_{0} \sin \alpha \sin \theta+y_{0} \cos \alpha \sin \theta-(a-b) \cos \theta}{b \cos \theta} .
$$

\section{Multilayered Recurrent Network Model}

We consider a three-layered network (see Figure 2) that we will train with a supervised learning algorithm. The projected motion field (the "retina") is discretized into $13 \times 13$ pixels. We take $a=8$ and $b=30$ so that the input to the network spans a window $15.5 \times 15.5$ degrees of visual angle. To each pixel corresponds an input unit with an (idealized) speed-tuning characteristic, as shown in Figure 3. The output units of the network code for the slant and tilt of the moving plane. The hidden units represent the MT neurons. They receive input from the input units and from other hidden units, and relay their output activations to the output units. Since our MT neurons receive direct input from the speed-tuned input units, their CRFs are endowed with this type of speed selectivity. The bandwidth of our tuning curve corresponds to that of the tuned MT cells recorded experimentally (Lagae et al., 1993). In any case, the speed selectivity of 58 out of 147 MT cells reported in Lagae et al. (1993) belongs to this (prevailing) type. We also assume that the hidden units are direction selective, with their preferred direction along the y-axis (see Figure 1), so that we can confine ourselves to translational motion along the y-axis only.

The hidden units are arranged in a $7 \times 7$ lattice. The output functions of these units are sigmoids (including a bias term). The connection weights from the input layer to each hidden unit are initialized with a connection kernel, that is, a smooth, decreasing function of the distance in projection plane coordinates. This is done in order to constrain the learning process so that it will preferentially develop localized CRFs with no motion gradient 


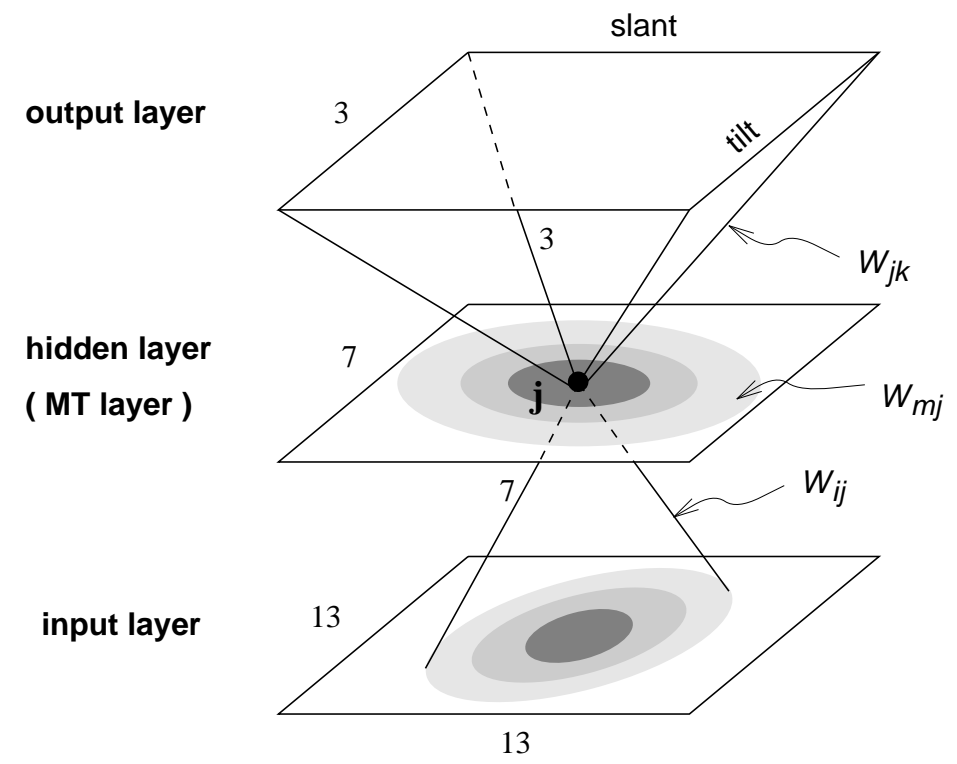

Figure 2: General outline of the three-layered network model. The gray-shaded ellipses represent the gaussian inter- and intralayer connection kernels of hidden unit $j$, prior to learning.

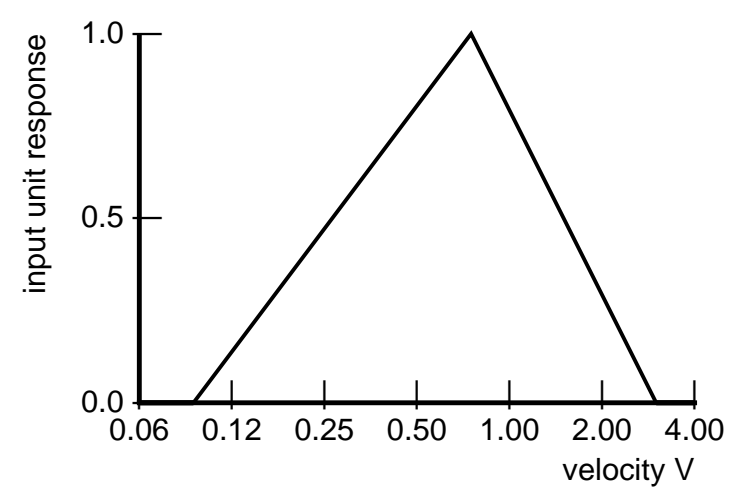

Figure 3: Idealized speed-tuning curve of an input unit. The response is plotted as a function of the velocity $V$ present at the retinal coordinate (projected motion field coordinate) represented by the unit. The preferred (optimal) velocity $V_{\text {opt }}=$ 0.75 and the lowest and highest velocities are 3/32 and 3, respectively (plotted on a log scale). Both the velocity and response scales are expressed in arbitrary units, without loss of generality. 
tuning of their own. ${ }^{1}$ For this localized connection kernel, we have used an elliptical gaussian since the great majority of MT cells have elliptical CRFs (Raiguel et al., 1995). The weights connecting the input units to a hidden unit are initialized according to a rotated elliptical gaussian connection kernel:

$$
W_{i j}=\exp \left(-\frac{x_{r}^{2}}{A^{2}}-\frac{y_{r}^{2}}{B^{2}}\right),
$$

with $W_{i j}$ the weight from input unit $i$ to hidden unit $j, A$ and $B$ the halflengths of the long and short axes of the ellipse, and $x_{r}$ and $y_{r}$ the rotated projection plane coordinates, rotated over an angle $\omega$ in the projection plane:

$$
\left[\begin{array}{l}
x_{r} \\
y_{r}
\end{array}\right]=\left[\begin{array}{cc}
\cos \omega & \sin \omega \\
-\sin \omega & \cos \omega
\end{array}\right] \cdot\left[\begin{array}{l}
x \\
y
\end{array}\right]
$$

with $x$ and $y$ the horizontal and vertical relative projection plane coordinates, relative with respect to the center of unit $j$ 's CRF. The long and short axes are chosen randomly within the intervals $[3,4.5)$ and $[1.5,3)$, respectively (expressed in input layer coordinates); the rotation angle $\omega$ is chosen randomly within $[0, \pi)$.

Each hidden unit also receives input from all other hidden units (see Figure 2). The intralayer connection weights are defined by a negative, circularsymmetrical gaussian kernel, modulated by the unit's activation:

$$
W_{m j}=-\exp \left(-\frac{x^{2}}{\sigma^{2}}\right) \cdot U_{j},
$$

with $W_{m j}$ the connection weight hidden unit $m$ makes with hidden unit $j$, $x$ the Euclidean distance in hidden-layer coordinates, $\sigma$ the radius of the gaussian, and $U_{j}$ the current activation of unit $j$. The last is required in order to ensure that the surround will reveal its presence only by the effect on the CRF response. ${ }^{2}$ Note that by these intralayer connections, the hidden units

\footnotetext{
${ }^{1}$ Treue and Andersen (1993) reported that approximately one-third of the MT neurons display a weak motion gradient tuning, but the stimuli they used were confined to the CRF only. Recently, Orban and coworkers have conducted a detailed analysis in macaque area MT, which clearly shows that motion gradient tuning does not arise from the CRF but rather from the asymmetric surround (Xiao et al., 1997). Hence, in our model, we want to examine the role of the surround in motion gradient tuning, and not that of the CRF.

${ }^{2}$ By this requirement, the hidden units become highly nonlinear and hence we cannot consider the newer (unsupervised) learning algorithms for developing localized RFs (Olshausen \& Field, 1996, p. 609). These algorithms assume linear response properties and work well when modeling simple cells in the primary visual cortex; however, it remains an open question how these algorithms could provide an account of other, more complex and nonlinear response properties of neurons at later stages in the visual pathway. Hence, by the nonlinear center-surround interaction in the MT cells and by the restriction that the CRF itself may not display any motion gradient tuning (see footnote 1), we have opted for an initialization with localized connection kernels for the CRF and the surround separately.
} 
form a recurrent network. In order to avoid boundary effects, we assume a toroidal intralayer connection pattern.

Finally, each hidden unit projects to all output units. The 9 output units have linear output functions and code for nine slant-tilt combinations (in degrees): 60/0, 0/0,60/180,60/45,60/90,60/135, 60/315, 60/270, and $60 / 225$, respectively. The network is trained with a modified backpropagation algorithm operating in batch mode (see the Appendix); the radius $\sigma$ in equation 3.3 is determined by cross-validation in steps of 0.05 (best obtained result is for $\sigma=0.25$ ). We consider two speeds at which the plane moves, $V_{p}=0.5$ and $1 .^{3}$ The training set is obtained by calculating, for the nine slant and tilt combinations, the corresponding projected motion fields using equation 2.3. From these motion fields, the output of each input unit is calculated according to its speed-tuning characteristic (see Figure 3). For a given slant-tilt combination, the desired output of the network is 1 for the corresponding output unit and 0 for all other units (1-out-of- $N$ coding). One epoch consists of applying each training pattern 100 times, and learning continues until the mean squared training error is below $10^{-2}$ or slowly decreasing. Typically, about 50,000 training epochs are needed.

\section{Simulation Results}

Here we focus on the response properties of the hidden units and map their RFs in a manner similar to single-cell recording. In order to map the CRF, we apply a localized velocity stimulus $V_{\text {opt }}$ (one pixel in size), which optimally activates the corresponding input unit, and take the response of the hidden unit as the magnitude of its CRF at that location (in projection plane coordinates). We repeat this procedure for all input units in order to obtain the hidden unit's CRF. In order to map the surround, we first apply a localized velocity stimulus $V_{\text {opt }}$ to the input unit corresponding to the center of the CRF and denote the hidden unit's response as $R_{C}$. We then choose an additional (a different) input unit, apply to it a localized velocity stimulus $V_{\text {opt }}$, and denote the response of the hidden unit to both stimuli as $R_{C+S}$. This response results from the interaction between CRF and surround and is assigned to the position of the additional input unit (in projection plane coordinates). The neurophysiologists then consider the net contribution from the surround as the difference $R_{C+S}-R_{C}$ (Raiguel et al., 1995). We repeat the previous procedure for all input units different from the one representing the CRF center.

After the connection weights are initialized, the network is run until the average squared error between the activity of the output units, averaged for the nine slant-tilt combinations, and the desired activities for these units

\footnotetext{
${ }^{3}$ We limit the speed range also since the scaling factor in our SFM problem depends on $V_{p}$.
} 

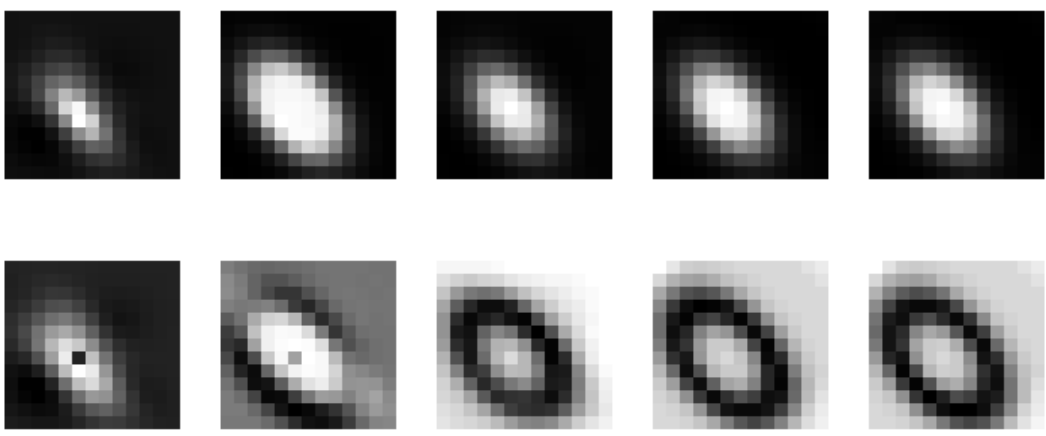

Figure 4: Evolution of the CRF (top) and the surround (bottom) map of hidden unit 31 during training. From left to right: The CRFs and the surrounds at 0 (initialization), 300, 4500, 23,123, and 46,035 epochs (convergence). The CRF and surround maps are represented by gray levels. For the sake of representation, the CRF maps are normalized between 0 (black) and 1 (white); the surround maps are normalized between their minimal (negative) value (black) and maximal value (white). The response range represented by the initial surround map is at least 5 to 10 times smaller than that of the other surround maps. Note that, by definition, the position of the CRF center in each surround map always corresponds to a zero response value (cf. the isolated pixel in the initial surround map).

is lower than $10^{-2}$. This is achieved after 46,035 training epochs. The output units are active only for a particular slant-tilt combination (1-out-of- $N$ coding), but it is clear that these units could have been trained to represent any other type of coding or slant-tilt tuning curve. Figure 4 depicts the evolution of the CRF and the surround of a hidden unit during training; the other hidden units undergo a similar evolution. This shows that although the CRFs and intralayer connection kernels are initialized with gaussians, the learning algorithm continues to change the connection weights until the desired slant-tilt selectivity is achieved at the output stage. From the CRFs and surrounds of all hidden units obtained at convergence, and for which a selection of surrounds is shown in the first column of Figure 6, the following observations can be made:

1. The CRFs obtained are all excitatory and correspond almost completely to the spatial layout of the forward connections. Furthermore, the CRFs do not display an internal structure that could favor slant detection (see footnote 1). Their shapes range from radially symmetrical to strongly elliptical.

2. The surrounds are predominantly antagonistic and, for most hidden units, the surround inhibition is confined to restricted regions on op- 


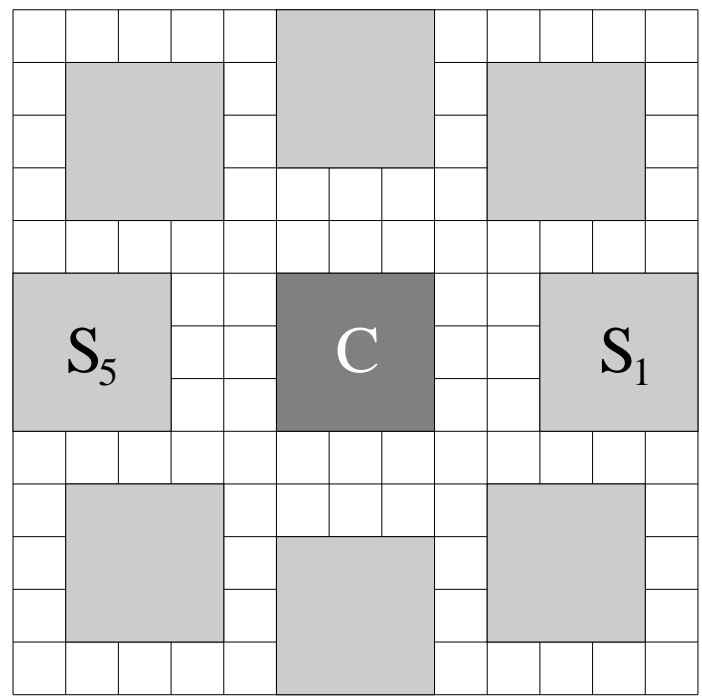

Figure 5: Motion field configuration used in the surround asymmetry tests (SAT1 and SAT2). Nine patches of $3 \times 3$ pixels are shown: one central patch (C) and eight satellite patches, of which two are labeled $\left(S_{1}, S_{5}\right)$. The gray shading filling these patches is an indication for the uniform speed applied in the corresponding motion fields (lighter shadings correspond to higher speeds).

posite sides (unit 43 in Figure 6) or on one side of the CRF (unit 46). These surround types are called bilaterally symmetric and asymmetric, respectively. Others display a more radially symmetric surround (unit 26) or a slightly more elongated one (unit 31). In addition, the axis connecting the inhibitory blobs of the bilaterally symmetric surround tends to be orthogonal to the long axis of the elliptically shaped CRF.

In order to classify the surrounds, we adopt the same procedure used for classifying experimentally recorded MT cells (Xiao et al., 1995). First, the surround asymmetry test (SAT) is applied to test eight satellite patches arranged symmetrically around the CRF center (see Figure 5). Patch C is centered on the CRF center and is stimulated uniformly at the optimal speed ( $V_{\text {opt }}$ in Figure 3 ). The response to patch $C$ is recorded and used as a reference for determining the degree of inhibition obtained when stimulated both the central patch and one of the satellite patches (SAT1) or two opposite satellite patches at the same time (e.g., $S_{1}, S_{5}$ ) (SAT2). The degree of inhibition elicited by each stimulus is then plotted in polar coordinates (second column in Figure 6). Second, for each SAT1 and SAT2 plot, the ratio between the strongest and weakest degree of inhibition is determined. If this ratio 
Table 1: Proportion of Surround Types Found for the Model (49 units) versus Area MT (86 cells).

\begin{tabular}{llll}
\hline & RS & \multicolumn{1}{c}{ BS } & \multicolumn{1}{c}{ A } \\
\hline Model & $18 \%$ & $29 \%$ & $53 \%$ \\
Area MT & 23 & 32 & 45 \\
\hline
\end{tabular}

Note: Radially symmetric (RS), bilaterally symmetric (BS), and asymmetric (A).

is smaller than 2:1 for both the SAT1 and SAT2 plots, then the surround is classified as radially symmetric; otherwise, the surround is considered to be heterogeneous. In the latter case, if the largest ratio is obtained for the SAT1 plot, then the surround is classified as asymmetric; otherwise, it is classified as bilaterally symmetric. The result of this classification is listed in Table 1. In the experimental results described in Xiao et al. (1995), the surrounds of 86 MT cells were classified as indicated in Table 1. Furthermore, the axis of the bilateral symmetric surrounds of these cells tends to be orthogonal to the long axis of the elliptically shaped CRF. Hence, the surround types obtained with our model are in accordance with the experimental evidence.

In order to show that the surrounds are speed-tuned, we apply a test procedure similar to the one used by Tanaka and coworkers (1986) for monitoring the speed-tuning of the surround inhibition in MT cells. A patch sized $3 \times 3$ pixels is centered on the CRF center and stimulated uniformly at the hidden unit's optimal speed $\left(V_{\text {opt }}\right)$. The remaining part of the motion field (retina) is stimulated uniformly at lower or higher speeds. The ratio of the response magnitude elicited by the combined CRF and surround stimulation is then divided by the response to the CRF alone. The resulting tuning curves for all hidden units were observed to belong to three classes (see the third column of Figure 6): the $\mathrm{V}(\mathrm{U})$-shaped tuning curve (units 26 and 43), the wedge-shaped tuning curve (unit 46), and the monotonically decreasing tuning curve (unit 31). Except for the wedge-shaped curve, which to some extent resembles the monotonically decreasing tuning curve, the tuning curves obtained correspond to the ones described by Tanaka and coworkers.

Finally, we determine the tilt tuning curves of our hidden units and parameterize them with respect to the slant. We apply to the network the projected motion fields corresponding to a slanted and tilted surface moving at speed $V_{p}=1$ and record the hidden unit's response magnitude (last column in Figure 6). The tilt tuning curves are largely in accordance with what is expected from the SATs: a larger degree of inhibition at a certain tilt angle usually corresponds to a lower response magnitude in the tilt tuning curve at the same angle (except for the tilt tuning curve for slant $\theta=0$ degree, which is always circular, by definition). The correspondence 
need not be perfect because of the differences in the techniques used for measuring the SATs and the tilt tuning curves. In order to verify if the tilt angle of the moving plane can be predicted from the SATs (Xiao et al., 1997), we have plotted, for the units with an asymmetric surround, the average tilt direction of the tilt tuning curves and the average direction of inhibition in the SATs (see the caption of Figure 6). In the ideal case, both vectors should point to opposite directions, which is the case for unit 31 but much less for unit 46. This behavior is also observed with actual MT cells (Xiao et al., 1997).

\section{Discussion}

Since the majority of the MT cells display a surround that envelops the classical receptive field, the presence of this surround and its role has received
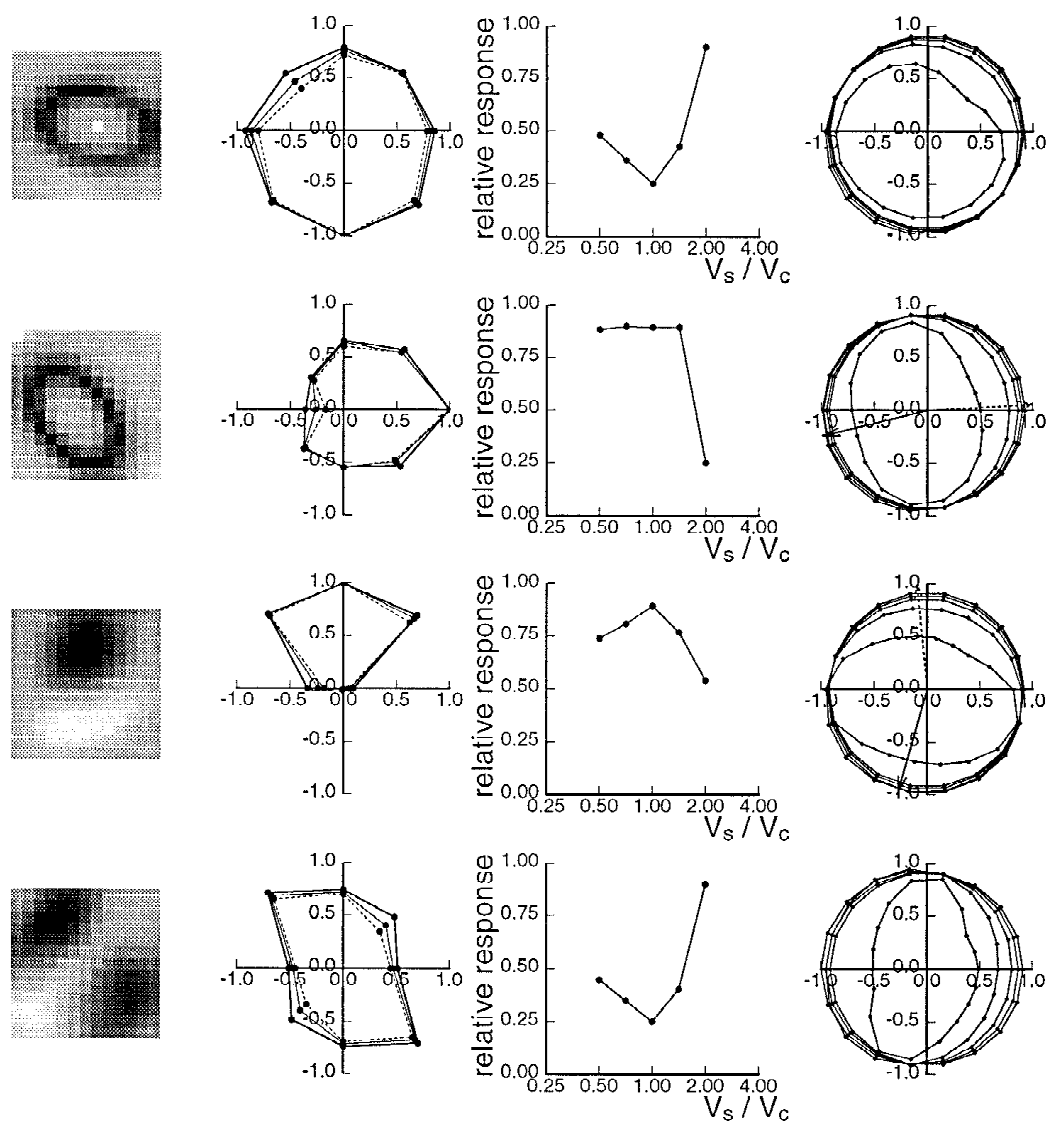
much attention in the neuroscience community. In this article, we have addressed the putative role of MT surrounds by developing a computational model with a clear purpose in mind. A three-layered neural network was trained to represent the slant and tilt of a translating flat surface from its projected motion vectors. The training procedure was used solely as a technique for developing the network and was not intended to be a model of learning in MT. The surrounds of the hidden units emerged through the training procedure as appropriate for extracting slant and tilt. Our model therefore has a direct parallel with the neural network model of Lehky and Sejnowski (1988) for extracting shape from shading (SFS). Their network was trained to compute curvature of Lambertian surfaces using only shading information. The hidden units developed RFs similar to those of simple cells in the primary visual cortex. This differs from our model in that the

Figure 6: Facing page. Surround maps and motion processing properties of hidden units 26, 31, 46, and 43 at convergence (row-wise, from top to bottom).

First column: The surround maps of the four hidden units. Based on the SAT plots (second column) the surrounds are classified as radially symmetric (unit 26), asymmetric (units 31 and 46), and bilaterally symmetric (unit 43). Note that the surround map of unit 31 corresponds to the right-most one in Figure 4 . The surround map conventions are as in Figure 4.

Second column: The SAT1 plots (except for unit 43) and SAT2 plot (unit 43). The SAT2 plot is shown since this test was decisive in classifying unit 43 . The SAT plots are shown for three satellite patch speeds: $V=0.75$ (thick full line), 1 (thin full line), and 1.125 (dashed line). The plots are normalized to unit length radius with respect to the maximal radius obtained for $V=0.75$, the unit's optimal speed $V_{\text {opt }}$. The origin represents zero inhibition.

Third column: The speed-tuning curves of the surround inhibition plotted (on a $\log$ scale) for different ratios between the surround speed $V_{s}$ and the CRF speed $V_{c}$, which is always $V_{\text {opt }}$. The curves are normalized with respect to the CRF response alone, without stimulating the surround.

Fourth column: The tilt tuning curves. Each set of curves represents the unit's response plotted in polar coordinates in steps of 20-degree tilt angle. The curves shown are parameterized with respect to five slant angles $\theta=0,20, \ldots, 80$ degrees and normalized with respect to the largest response vector in the set. The curve for $\theta=0$ degree is a circle, by definition, and the other curves become progressively less circular and more restricted in size when $\theta$ increases. For the sake of exposition, the tilt angle is defined as the angle between the direction of increasing speed gradient and the horizontal axis. For units 31 and 46 only: The full line arrow represents the average tilt direction, defined as the vector sum of all response vectors taken over all tilt and slant angles shown. Similarly, the dashed arrow represents the average direction of inhibition, defined as the vector sum of all degree of inhibition vectors shown in the corresponding SAT plots. The result of vector summation is then normalized to unit length. 
output units of the SFS model had biological counterparts as well. As far as we know, the output units in our case do not have biological counterparts, but it is clear that they can be endowed with slant-tilt tuning characteristics other than those assumed (i.e., a 1-out-of- $N$ coding).

Single-cell recordings show that $95 \%$ of the MT neurons exhibit a marked directional selectivity (Zeki, 1974; Maunsell \& Van Essen, 1983; Albright, 1984). Furthermore, MT has been shown to contain a systematic columnar arrangement of directionally selective neurons (Albright, Dessimone, \& Gross, 1984). All model neurons we have developed are assumed to belong to the same direction column and to be stimulated along their preferred direction of motion. Hence, since we do not have to consider any direction of motion in the input units, the overall network structure is greatly simplified and the training time dramatically reduced.

In the presence of a rotational component in the motion of the slanted plane, for example, around the line of sight, the projected motion vectors are a function of both the slant and the rotational velocity of the plane. Since MT cells respond to rotation-induced motion vectors, but without being rotationally selective (Lagae et al., 1994), the individual MT cell's response confounds slant- and rotation-induced motion stimuli. In our model, the MT neurons are also prone to this confusion; in addition, since the purpose of the model was to develop (i.e., to train) slant- and-tilt selective units from the gradient of the projected motion vectors, we had to confine ourselves to translational motion. This is a limitation of the training procedure, which was, as we said, not intended to be a model of learning in MT.

Raiguel et al. (1995) studied the spatial organization of RFs in area MT of anesthetized and paralyzed macaque monkeys. The size and shape of the RF was found by fitting with a generalized gaussian. Most of these RFs are elongated, and the axis of elongation tends to be orthogonal to the preferred direction of motion. The extent of the surround at half-height equaled, on average, three to four times that of the CRF. Raiguel et al. then suggested that antagonistic surrounds are constructed in MT, most likely through horizontal connections. The model developed in this article also contains horizontal connections for the MT units, and the antagonistic surrounds obtained are in accordance with those found experimentally. Hence, our model supports the hypothesis about the putative origin of the antagonistic surrounds.

Finally, although the hidden units in our network model are broadly tuned, only one additional (output) layer, with linear units and linear connections, suffices to recover the slant and tilt of a translating plane. Hence, we conjecture that populations of MT cells, with different surround types, can code linearly for slant and tilt of translating planes.

\section{Appendix: Learning Algorithm}

$/ *$

Let $\left\{W_{i j}\right\},\left\{W_{m j}\right\}$, be the set of hidden unit $j$ 's forward and 
feedback (intralayer) connections;

Let $\left\{W_{j k}\right\}$ be the set of output unit $k^{\prime}$ s forward connections;

Let $M$ be the number of training (input) patterns;

Let $V^{\mu}$ be training pattern $\mu$ determined using equation 2.3 and Figure 3;

Let $U_{j}$ be hidden unit $j^{\prime}$ s output activation;

Let $E$ be the training error

*/

/* Initialization */

Initialize $\left\{W_{i j}\right\}$ using equation 3.1, and $\left\{W_{m j}\right\}$ using equation 3.3 given $\sigma$

Initialize $\left\{W_{j k}\right\}$ randomly within the interval $[0,1)$

Set learning rate $\eta \leftarrow 0.3$

Set training error value $E_{\text {past }}>>$

/*

Backpropagation-through-time algorithm (Rumelhart, Hinton, \& Williams, 1986), operating in batch mode, and modified:

1) to encompass weighted and modulated intralayer connections $W_{m j}\left(U_{j}\right)$ equation 3.3; *1

2) to speed up training time by modifying $\eta$ and by adding noise to it

Do until training error $E<10^{-2}$ or slowly decreasing

\{

Set $\left\{\left\{\Delta W_{i j} \leftarrow 0, \forall i \in\right.\right.$ input layer $\}, \forall j \in$

hidden layer $\} ;\left\{\left\{\Delta W_{j k} \leftarrow 0, \forall j \in\right.\right.$ hidden layer $\}, \forall k \in$ output layer $\}$

Do 100 times /* the size of one epoch */

\{

Do $\mu=1$ to $M \quad{ }^{*} \forall$ training patterns */

\{

Take input pattern $V^{\mu}$

Propagate activity from input to hidden layer

Calculate initial activations of hidden neurons; $U_{j}, \forall j \in$ hidden layer

Propagate $U_{j}, \forall j$, back to hidden units, through $W_{m j}\left(U_{j}\right)$ equation 3.3

Calculate new activations of hidden neurons; $U_{j}, \forall j$

Propagate activity from hidden to output layer

/* Backprop step */

Determine output and hidden unit training errors and:

Do $\forall j \in$ hidden layer

\{

$\Delta W_{i j} \leftarrow \Delta W_{i j}-\eta \frac{\partial E}{\partial W_{i j}}, \forall i \in$ input layer

$\Delta W_{j k} \leftarrow \Delta W_{j k}-\eta \frac{\partial E}{\partial W_{j k}}, \forall k \in$ output layer

\} 


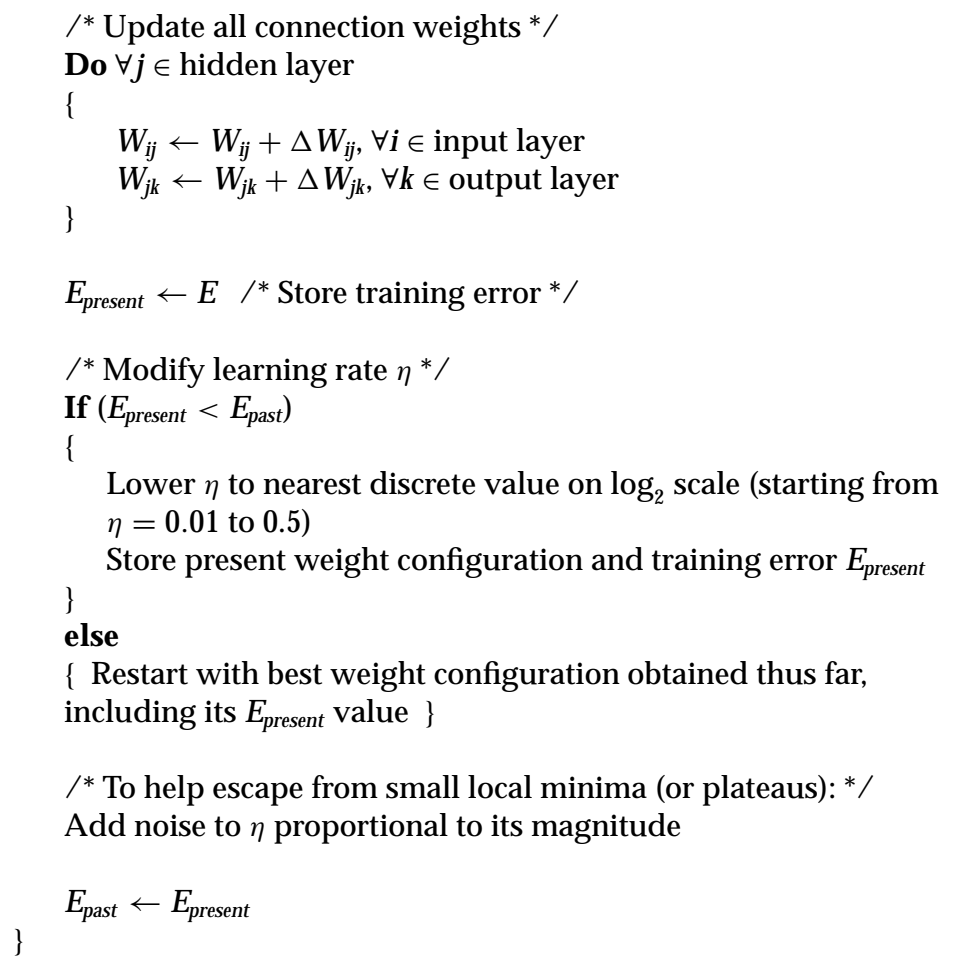

\section{Acknowledgments}

We express our gratitude to D.-K. Xiao, Laboratorium voor Neuro- en Psychofysiologie, K. U. Leuven, whose input was indispensable in shaping the model. We also thank G. A. Orban and S. Raiguel, of the same laboratory, for helpful discussions, and H.-J. Sun, Institute of Biophysics, Academia Sinica, Beijing, for his help in developing the learning algorithm. The first author is supported by a postdoctoral grant received from the Research Fund of the K. U. Leuven (F/95/138). The second author is a research associate of the Fund for Scientific Research-Flanders (Belgium) and is supported by research grants received from the Fund for Scientific Research (G.0185.96) and the European Commission (ECVnet EP8212).

\section{References}

Albright, T. D. (1984). Direction and orientation selectivity of neurons in visual area MT of the macaque. J. Neurophysiol., 52, 1106-1130.

Albright, T. D. (1993). Cortical processing of visual motion and its role in the stabilization of gaze. In F. A. Miles \& J. Wallman (Eds.), Visual motion and its role in the stabilization of gaze (pp. 177-201). Amsterdam: Elsevier. 
Albright, T. D., Desimone, R., \& Gross, C. G. (1984). Columnar organization of directionally selective cells in visual area MT of the macaque. J. Neurophysiol., 51, 16-31.

Allman, J. M., \& Kaas, J. H. (1971). A representation of the visual field in the caudal third of the middle temporal gyrus of the owl monkey (Aotus trivirgatus). Brain Res., 31, 85-105.

Allman, J., Miezin, F., \& McGuinness, E. (1985). Direction- and velocity-specific responses from beyond the classical receptive field in the middle temporal are (MT). Perception, 14, 105-126.

Andersen, R. A., \& Siegel, R. M. (1986). Two- and three-dimensional structure from motion sensitivity in monkeys and humans. Soc. Neurosci. Abstr., 12, 1183.

Buračas, G. T., \& Albright, T. D. (1994). The role of MT neuron receptive field surrounds in computing object shape from velocity fields. Advances in Neural Information Processing Systems, 6, 969-976.

Buračas, G. T., \& Albright, T. D. (1996). Contribution of area MT to perception three-dimensional shape: A computational study. Vis. Res., 36, 869-888.

Droulez, J., \& Cornilleau-Pérès (1990). Visual perception of surface curvature: The spin variation and its physiological implementation. Biol. Cybern., 62, 211-224.

Dubner, R., \& Zeki, S. M. (1971). Response properties and receptive fields of cells in an anatomically defined region of the superior temporal sulcus in the monkey. Brain Res., 35, 528-532.

Hussain, M., Treue, S., \& Andersen, R. A. (1989). Surface interpolation in threedimensional structure-from-motion perception. Neural Computation, 1, 324333.

Koenderink, J. J., \& van Doorn, A. J. (1992). Second order optic flow. J. Opt. Soc. Am. A, 9, 530-538.

Lagae, L., Gulyás, B., Raiguel S., \& Orban, G. A. (1989). Laminar analysis of motion information processing in macaque V5. Brain Res., 496, 361-367.

Lagae, L., Maes, H., Raiguel S., Xiao, D.-K., \& Orban, G. A. (1994). Responses of macaque STS neurons to optic flow components: A comparison of areas MT and MST. J. Neurophysiol., 71, 1597-1626.

Lagae, L., Raiguel S., \& Orban, G. A. (1993). Speed and direction selectivity of macaque middle temporal neurons.J. Neurophysiol., 69, 19-39.

Lehky, S. R., \& Sejnowski, T. J. (1988). Network model of shape-from-shading: Neural function arises from both receptive and projective fields. Nature, 333, 452-454.

Maunsell, J. H. R., \& Van Essen, D. C. (1983). Functional properties of neurons in middle temporal visual area of the macaque monkey. I. Selectivity for stimulus direction. J. Neurophysiol., 49, 1127-1147.

Movshon, J. A., Adelson, E. H., Gizzi, M. S., \& Newsome, W. T. (1985). The analysis of moving visual patterns. In C. Chagass, R. Gattas, \& C. Gross (Eds.), Pattern recognition mechanisms (pp. 117-151). Heidelberg: Springer-Verlag.

Olshausen, B. A., \& Field, D. J. (1996). Emergence of simple-cell receptive field properties by learning a sparse code for natural images. Nature, 381, 607-609. 
Raiguel, S., Van Hulle, M. M., Xiao, D.-K., Marcar, V. L., \& Orban, G. A. (1995). Shape and spatial distribution of receptive fields and antagonistic motion surrounds in the middle temporal area (V5) of the macaque. Eur. J. Neurosci., 7, 2064-2082.

Rumelhart, D. E., Hinton, G. E., \& Williams, R. J. (1986). Learning internal representations by error propagation. In D. E. Rumelhart \& J.L. McClelland (Eds.), Parallel distributed processing (Vol. 1, pp. 318-362). Cambridge, MA: MIT Press.

Tanaka, K., Hikosaka, H., Saito, H., Yukie, Y., Fukada, Y., \& Iwai, E. (1986). Analysis of local wide-field movements in the superior temporal visual area of the macaque monkey. J. Neurosci., 6, 134-144.

Treue, S., \& Andersen, R. A. (1993). Tuning of MT cells to velocity gradients. Invest. Ophthalmol. Vis. Sci., 34, 813.

Treue, S., Andersen, R. A., Ando, H., \& Hildreth, E. C. (1995). Structure from motion: Perceptual evidence for surface interpolation. Vis. Res., 35, 139-148.

Xiao, D.-K., Marcar, V. L., Raiguel, S. E., \& Orban, G. A. (1997). Selectivity of macaque MT/V5 neurones for surface orientation in depth specified by motion. Eur. J. Neurosci., 9, 956-964.

Xiao, D.-K., Raiguel, S., Marcar, V., Koenderink, J., \& Orban, G. A. (1995). Spatial heterogeneity of inhibitory surrounds in the middle temporal visual area. Proc. Natl. Acad. Sci. USA., 92, 11303-11306.

Zeki, S. M. (1974). Functional organization of a visual area in the posterior bank of the superior temporal sulcus of the rhesus monkey. J. Physiol., 236, 549-573.

Received August 28, 1996; accepted May 6, 1997. 